\section{Migraine: incorrect self-management for a disabling disease}

\author{
Giovanna Viticchi, ${ }^{1}$ Lorenzo Falsetti, 2 \\ Marco Bartolini, ${ }^{1}$ Laura Buratti, ${ }^{1}$ \\ Alfio Ulissi, ${ }^{3}$ Maurizio Baldassari, ${ }^{3}$ \\ Leandro Provinciali, ${ }^{1}$ Mauro Silvestrini ${ }^{1}$ \\ 1Neurological Clinic, Marche \\ Polytechnic University, Ancona; \\ 2Internal and Subintensive Medicine, \\ Ospedali Riuniti Ancona; ${ }^{3}$ Occupational \\ Medicine Ward, Ospedali Riuniti \\ Ancona, Italy
}

\begin{abstract}
Migraine is an economically and socially relevant pathology but patients' consciousness and consequently a proper management is often unsatisfactory. In this study, we aimed to evaluate the disease awareness, the general approach and the impact on working activity in a population of headache-affected hospital workers. During an 18-month period, hospital workers responded to a questionnaire investigating headache presence, characteristics and awareness. Specific attention was paid to define headache impact on working activities. 1774 consecutive subjects were enrolled. Headache was documented in $45.7 \%$ of the sample, migraine in $16.6 \%$. $40.6 \%$ of the patients with headache and $50.7 \%$ of the migraneous subjects had a specialist medical evaluation. Triptans use was significantly lower than non-steroidal anti-inflammatory drugs use. $39.5 \%$ of migraneous complained of attacks during working time, $60.2 \%$ acknowledged a negative influence on work quality. $25.5 \%$ of migraneous patients reported a loss of working days attributable to the disease. Migraine is disabling for workers with negative consequences on job capacity and quality. Efforts should be made to improve disease awareness to stimulate a better diagnostic and therapeutic approach.
\end{abstract}

\section{Introduction}

The world health organization (WHO) established migraine as the $6^{\text {th }}$ cause of disability worldwide in 2015.1,2 In spite of this, awareness and specific knowledge about the disease is still unsatisfactory. ${ }^{3,4}$ The Eurolight Project showed that headache, particularly migraine, results in relevant direct and indirect costs. Among the indirect costs, loss of work productivity and absenteeism should deserve particular consideration. ${ }^{5}$ A recent review showed that the mean per-person annual cost for migraine is $€ 1222$ : indirect costs accounted for $93 \%$ divided by reduced productivity ( $€ 765)$ and absenteeism (€371). 6 It is important to underline that headache is a highly prevalent pathology, affecting about $15 \%$ of the global population: the large part of affected people is in the age range between 25 and 55 years, which represents the most relevant job-productive life period. ${ }^{7}$ Another relevant aspect is headache impact on quality of life. Affected patients tend to avoid social events, sport or holidays in order to reduce the risk of a painful attack. Some recent studies underlined that migraine could be defined a disabling illness 7,8 with a social impact comparable to that of other noncommunicable diseases such as chronic obstructive pulmonary disease (COPD), chronic kidney disease or chronic heart failure. Despite the impact of migraine on the different aspects of life, the self-consciousness of illness and the management turns out to be unsatisfactory: several studies report that only a negligible part of migraine patients refer to headache centres. ${ }^{9,10}$ Moreover, studies regarding the pharmacologic treatment have shown that specific therapies for the acute attack of migraine, namely triptans, are underused.11-13 Aim of this study was to investigate, in a population of hospital workers, the prevalence of headache and migraine, the management and the awareness of illness. Moreover, we investigated the working consequences associated to migraine and the correct use of specific treatments.

\section{Materials and Methods}

The study was performed at the University Hospital of Ancona. In an 18month period, we submitted a self-questionnaire regarding the presence, characteristics and impact of headache to all the hospitalworker categories. The questionnaire was completed during one of the periodic medical routine screening visits for the verification of the ability to maintain the job function. The questionnaire was organized in four sections: the first one investigated about the presence of headache attacks in the last three months, the age of symptom onset and the clinical characteristics of the attacks. The second section, to be completed only by subjects with a history of headache, asked them if they had ever referred to their general practitioner or to a neurologist or to a headache centre. The third section regarded the types of drugs
Correspondence: Lorenzo Falsetti, Internal and Subintensive Medicine, Ospedali Riuniti di Ancona, Via Conca 1, 60020 Ancona, Italy. Tel.:+39.071.596.4017 - Fax: +39.071.596.3215. E-mail: drfalsetti@yahoo.it

Key words: headache, migraine, burden, consciousness, disability

Contributions: GV, LB, MB, LP, AU, MB and MS participated to the concept of the study; $\mathrm{AU}$ and $\mathrm{MB}$ enrolled the patients and acquired the data; GV, LF, LB and MS analysed the study results and wrote the paper; LF conducted the statistical analysis; MS and LP supervised and revised the study.

Conflict of interest: the authors declare no conflict of interest.

Funding: none.

Received for publication: 15 December 2017. Accepted for publication: 22 January 2018.

This work is licensed under a Creative Commons Attribution NonCommercial 4.0 License (CC BY-NC 4.0).

(C) Copyright G. Viticchi et al., 2018

Licensee PAGEPress, Italy

Neurology International 2018; 10:7510

doi:10.4081/ni.2018.7510

employed for acute attacks. Finally, in the fourth section, we investigated the impact of headache on work quality and productivity. All subjects were consecutive without any admission bias; the questionnaire was completely anonymous, and the enrolled subjects completed it without a face-to-face contact with the examiner. For the diagnosis of migraine, the Italian version of IDmigraine test was used (Table 1). This is a validated three-question test to indirectly perform a diagnosis of migraine. ${ }^{12}$ A threequestion test to evaluate the impact of headache on the work activities was then elaborated (Table 2). Each subject gave an informed consent for the participation to the study. The local Ethics Committee approved the study protocol.

\section{Statistical analysis}

The type of drugs used and the category of workers were summarized as different categorical variables. The presence of headache and migraine was collected in different binary variables. The ID-Migraine test was collected as a single, dichotomous variable. The occurrence of migraine attacks, absence from work and reduced productivity were summarized as dichotomous variables. We adopted the $\chi^{2}$ test to investigate differences in the distribution of 
categorical or dichotomous variables. Statistics was performed with SPSS 13.0 for Windows systems.

\section{Results}

During the study period, we enrolled 1774 consecutive subjects. All the hospital job categories were included: physicians, nurses, technicians, administrative employees and sanitary workers. Epidemiological data are summarized in Table 3.

810 patients $(45.7 \%$ of the sample) suffered from headache. In this group, all the typologies of headache were considered. Tension-type headache and migraine were the most represented categories. The IDMigraine test identified migraine in $16.6 \%$ of the interviewed subjects (294 patients).

In the headache group, $40.6 \%$ of the affected subjects (329 patients) referred to their general practitioner or to a headache center; among the group of migraineurs, $50.7 \%$ (149 subjects) asked for these evaluations.

Among the 810 workers suffering from headache, $73.5 \%$ (635 subjects) did not take any medication for symptom control. When we analyzed the subgroup of patients affected by non-migraine headache, prevalence of subjects taking any kind of therapy for the acute attack was $13.2 \%$ (68 subjects), while among migraine patients the percentage of those who had at least one drug rose to $37.2 \%$ (109 subjects; $\mathrm{P}<0.0001$ ). We did not observe any significant difference among the classes of workers for the type of medication in the headache group $(\mathrm{P}=0.719)$ and, similarly, in migraine patients $(\mathrm{P}=0.370)$.

In the subgroup of migraneous patients, $185(62.9 \%)$ did not take any drug, 11 (3.74\%) used paracetamol, 25 (8.5\%) triptans, 49 (16.7\%) non-steroidal anti-inflammatory drugs (NSAIDS), 5 (1.7\%) steroids, while $1(0.34 \%)$ took ergotamine derivatives; the remaining part of this subpopulation (17 subjects, $5.78 \%$ ) took undefined analgesics. The use of triptans was significantly lower than that of NSAIDs. The distribution of the drugs use was significantly different $(\mathrm{P}<0.0001)$ at $\chi^{2}$ test. Results were particularly unexpected among migraineurs physicians (28 subjects, $9.5 \%$ of the migraine workers): during acute attacks, 19 $(67.9 \%)$ did not use any drug, 1 (3.57\%) took paracetamol, 3 (10.7\%) NSAIDS, 1 (3.57\%) unspecified analgesic drugs and 1 (3.57\%) still used ergotamine derivatives. Only 3 (10.7\%) doctors correctly used triptans. Even in this subgroup, we observed a significant difference $(\mathrm{P}<0.0001)$ in the dis- tribution of the use of the drugs at $\chi^{2}$ test.

178 patients $(22.0 \%)$ complained of headache attacks during working time. 266 (32.8\%) subjects declared that headache influenced work quality and $89(11.0 \%)$ acknowledged absence from work due to a headache attack. Among migraine workers, $116(39.5 \%)$ patients complained of attacks during work time. 177 patients $(60.2 \%)$ had a negative influence on work activities and in $75(25.5 \%)$, migraine attacks caused loss of working days.

\section{Discussion}

Our data show a remarkable impact of headache on work productivity and, more generically, on job quality. In fact, the large majority of patients affected by headache, particularly migrane, reported a relevant negative quantitative and qualitative influence of the illness on their work. $25.5 \%$ declared loss of at least a working day in the previous three months because of a migraine attack. These data are in accordance with the findings of different investigations showing a significant association between migraine and low productivity, difficulties and absenteeism. ${ }^{13,14} \mathrm{~A}$ recent Italian sub-study of the Eurolight Project

Table 1. The ID-Migraine Test.

\section{During the last 3 months, have you ever had the following with your headaches}

1 Have you ever felt nauseated or sick in your stomach during your headaches?

2 Did light bother you when you had a headache (a lot more than when you do not have headaches)?

3 Did your headache limit your ability to work, study or do what you needed to do for at least 1 day?

The cut-off point for a test-diagnosis of migraine headache was at least two positive responses.

Table 2. Questionnaire about hospital workers' headache.

\section{Fourth part: headache and work activities}

1 Are headache attacks frequent during your work activities?

2 Do headache attacks influence your work productivity?

3 Have you ever been absent from work because of headache?

Table 3. Epidemiological data.

\begin{tabular}{lccc} 
Variable & Overall & Headache & Migraine \\
Number & 1774 & 810 & 294 \\
Males, $\mathrm{n} .(\%)$ & $648(36.5)$ & $211(26.1)$ & $59(20.1)$ \\
\hline Age $( \pm$ SD) & $40.1 \pm 11.74$ & $39.7 \pm 11.07$ & $42.1 \pm 10.56$ \\
Referring to a General Practitioner, Neurologist, Headache center, n. (\%) & $337(19.0)$ & $329(40.6)$ & $149(50.7)$ \\
\hline Taking drugs for acute attacks, n. (\%) & $184(10.4)$ & $177(21.9)$ & $109(37.2)$ \\
Attacks during work activity, n. (\%) & $178(10)$ & $178(22)$ & $116(39.6)$ \\
\hline Attacks influence job quality, n. (\%) & $269(15.2)$ & $266(32.8)$ & $176(60.1)$ \\
Attacks force absence from job, n. (\%) & $93(5.2)$ & $89(11.0)$ & $74(25.3)$ \\
\hline
\end{tabular}


reported the loss of about 2.3 days from paid work and 2.4 days from household work in headache patients during a 3-month period. ${ }^{15}$ An extensive review regarding the relationship between migraine and workrelated activities showed that, on average, a migraine patient loses 3.5 working days per year and has reduced effectiveness for about 10 days. ${ }^{16}$ The greatest difficulties were observed in activities involving problemsolving and speaking performance. This kind of activities, although relevant for all work categories, can be considered as fundamental and essential for hospital workers. Accordingly, our findings should be considered very troubling since they refer to health workers who, theoretically, should exert a high level of attention and reasoning during their activities.

Another relevant aspect emerging from our investigation is the very low awareness of hospital workers to a very relevant health problem. This aspect has already been underlined by different investigations showing low sensibility about self-health problems in hospital workers. In particular, a previous study of our group compared a sample of hospital workers with a general population sample showing that in-hospital workers had a longer diagnostic delay with respect to that of outpatients (14.89 years vs. 12.13 years). ${ }^{17}$ Moreover, when we analysed the single job category, we found that no difference was present between clinical (physicians, nurses) and non-clinical categories. All these data underline a commonly uncorrected management of migraine by hospital workers, especially clinicians with potential very serious consequences.

In literature many data underline the burden of headache on the quality of life, while fewer studies approach the impact of this disease on working capacity. Very few data are also available regarding the selfawareness of illness, and highlight that only specialist medical consultation could increase the subject's self-awareness of migraine and could indicate the employment of appropriate medications for the treatment of acute attacks. 18

In our study, only $50.7 \%$ of people affected by headache had a general practitioner, a neurologist or a headache centre consultation. According to literature data, about $30 \%$ of migraineurs do not have any medical check-up for their headache in their life, and more than $20 \%$ do not consult a physician for at least one year. ${ }^{19}$ These data reflect how such a pathology can be considered not alarming but is disabling for the affected subjects.

The possible explanations for this apparent paradox can be various. First, the frequent familiarity for migraine can deter- mine a sort of habituation to this pathology and to the several, relevant symptoms associated to pain like nausea, vomiting, and photophobia. In the past, our group largely investigated the diagnostic delay in migraine with and without aura, showing that people can wait up to six years on average to obtain a correct diagnosis. $3,4,20$

Second, often patients or physicians formulate a wrong diagnosis. Subjects tend to associate headaches to sinusitis or cervical spine pathologies. As a result, they take unspecific therapies for years, or undergo useless and expensive radiological or laboratory exams. ${ }^{3,21}$ Finally, there is a general opinion that headache is a not relevant pathology, probably because it is very diffused and usually benign. Also health-care professionals define headache as a trivial complaint. ${ }^{7}$ Despite the common sense, many studies have shown that headache significantly compromises the quality of life. ${ }^{7,22}$

Data regarding the correct use of specific therapies are discouraging. Only a minor part of the investigated subjects considered triptans for migraine attacks. The larger part of our sample preferred to avoid the intake of any drug. This finding is in accordance with the current literature data showing that the use of triptans is very low worldwide in spite of their good tolerability and high specificity of the therapeutic effect.11,23 In addition, the percentage of discontinuers is relevant. ${ }^{12}$ Recent data have shown that the utilization of triptans in the general population varies between 3.0 and $19 \% .{ }^{11,24} \mathrm{~A}$ recent study about headache in in-hospital workers showed that the $22 \%$ of them used prescription drugs versus more than $56 \%$ that used over-the-counter drugs. ${ }^{24}$ Despite these data, some studies found that the regular use of triptans could significantly improve the productivity of workers, ${ }^{16}$ increase work efficiency 25,26 and reduce psychological symptoms associated to migraine, such as depression and anxiety. 15,27

Migraine can cause social disability for its relevant impact on the quality of life. Recently, Leonardi and colleagues defined migraine as a stigma like other pathologies such as epilepsy. ${ }^{28}$ Several studies have shown a narrow relationship between headache and depression, anxiety or, in particular cases, suicide. ${ }^{29}$ Migraineurs often present with a sense of inadequacy and fear of migraine attacks. ${ }^{13}$ Disability could have a multi-dimensional approach because according to WHO classification of functioning, disability and health, it is unethical to consider the impact of headache only from an economic point of view. Human relevance of sufferance and impact on the quality of life and the presence of chronic comorbidities should be carefully considered. 7,30

\section{Conclusions}

In conclusion, our data confirm that headache, particularly migraine, can be considered as a disabling barrier with social and economic consequences that should be prevented and treated, especially by improving a correct clinical and therapeutic management. In the attempt to reduce the burden of this disease, knowledge and selfconsciousness should be stimulated through information and health education.

\section{References}

1. Global Burden of Disease Study 2013 Collaborators. Global, regional, and national incidence, prevalence, and years lived with disability for 301 acute and chronic diseases and injuries in 188 countries, 1990-2013: a systematic analysis for the Global Burden of Disease Study 2013. Lancet 2015; 386:743-800.

2. Steiner TJ, Stovner LJ, Katsarava Z, et al. The impact of headache in Europe: principal results of the Eurolight project. J Headache Pain 2014;21:15-31.

3. Viticchi G, Silvestrini M, Falsetti L, et al. Time delay from onset to diagnosis of migraine. Headache 2011;51:232-6.

4. Viticchi G, Bartolini M, Falsetti L, et al. Instrumental exams performance can be a contributing factor to the delay in diagnosis of migraine. Eur Neurol 2014;71:120-5.

5. Linde M, Gustavsson A, Stovner LJ, et al. The cost of headache disorders in Europe: the Eurolight project. Eur J Neurol 2012;19:703-11.

6. Lipton RB, Stewart WF, von Korff M. Burden of migraine: societal costs and therapeutic opportunities. Neurology 1997;48:S4-9.

7. Leonardi M, Steiner TJ, Scher AT, Lipton RB. The global burden of migraine: measuring disability in headache disorders with WHO's classification of functioning, disability and health (ICF). J Headache Pain 2005; 6:429-40

8. D'Amico D, Grazzi L, Usai S, et al. Disability in chronic daily headache: state of the art and future directions. Neurol Sci 2011;32:S71-6.

9. Cevoli S, D'Amico D, Martelletti P, et al. Underdiagnosis and undertreatment 
of migraine in Italy: a survey of patients attending for the first time 10 headache centres. Cephalalgia 2009;29:1285-93.

10. Viticchi G, Falsetti L, Buratti L, et al. Triptan use among hospital workers affected by migraine. Neurol Sci 2015;6:157-9.

11. Panconesi A, Pavone E, Franchini M, et al. Triptans: low utilization and high turnover in the general population. Cephalalgia 2010;30:576-81.

12. Cousins G, Hijazze S, Van de Laar FA, Fahey T. Diagnostic accuracy of the ID Migraine: a systematic review and meta-analysis. Headache 2011;51:11408.

13. Raggi A, Giovannetti AM, Quintas R, et al. A systematic review of the psychosocial difficulties relevant to patients with migraine. J Headache Pain 2012;13:595-606.

14. D'Amico D, Grazzi L, Curone M, et al. Difficulties in work activities and the pervasive effect over disability in patients with episodic and chronic migraine. Neurol Sci 2015;36:9-11.

15. Allena M, Steiner TJ, Sances G, et al. Impact of headache disorders in Italy and the public-health and policy implications: a population-based study within the Eurolight Project. J Headache Pain 2015;16:100.

16. Raggi A, Covelli V, Leonardi M, et al.
Difficulties in work-related activities among migraineurs are scarcely collected: results from a literature review. Neurol Sci 2014;35:23-6.

17. Viticchi G, Falsetti L, Pettinari P, et al. Headache in a population of hospital workers. Neurol Sci 2014;35:157-8.

18. Lucas C, Géraud G, Valade D, et al. Recognition and therapeutic management of migraine in 2004, in France: results of FRAMIG 3, a French nationwide population-based survey. Headache 2006;46:715-25.

19. Lipton RB, Scher AI, Kolodner K, et al. Migraine in the United States: Epidemiology and patterns of health care use. Neurology 2002;58:885-94.

20. Viticchi G, Bartolini M, Falsetti L, et al. Diagnostic delay in migraine with aura. Neurol Sci 2013;34:S141-2.

21. Viticchi G, Silvestrini M, Falsetti L, et al. The role of instrumental examinations in delayed migraine diagnosis. Neurol Sci 2011;32:S143-4.

22. Stovner LJ, Hagen K, Jensen R, et al. The global burden of headache: a documentation of headache prevalence and disability worldwide. Cephalalgia 2007;27:193-210.

23. Wu J, Hughes MD, Hudson MF, Wagner PJ. Antimigraine medication use and associated health care costs in employed patients. J Headache Pain
2012;13:121-7.

24. Da Cas R, Nigro A, Terrazzino S, et al. Triptan use in Italy: Insights from administrative databases. Cephalalgia 2015;35:619-26.

25. Hughes MD, Wu J, Williams TC, et al. The Experience of Headaches in Health Care Workers: Opportunity for Care Improvement. Headache 2013;53:9629.

26. Silberstein SD, Cady RK, Sheftell FD, et al. Efficacy of eletriptan in migrainerelated functional impairment: functional and work productivity outcomes. Headache 2007;47:673-82.

27. Lainez MJ, Lopez A, Pascual AM. Effects on productivity and quality of life of rizatriptan for acute migraine: a workplace study. Headache 2005;45: 883-90.

28. Leonardi M, Raggi A, Grazzi L, D'Amico D. Disability, ICF biopsychosocial model and burden of migraine. J Headache Pain 2015; 16:A2.

29. Nović A, Kõlves K, O'Dwyer S, Leo D. Migraine and Suicidal Behaviours: A Systematic Literature Review. Clin J Pain 2016;32:351-64.

30. Negro A, D'Alonzo L, Martelletti P. Chronic migraine: comorbidities, risk factors, and rehabilitation. Intern Emerg Med 2010;5:S13-9. 